Sir,

\section{Vascularised epiretinal membrane associated with vasoproliferative tumour}

Retinal vasoproliferative tumour (VPT) is a benign entity known to be associated with epiretinal membrane (ERM) formation. The suspicion that some VPT-related membranes have vascular elements, based on clinical fundoscopic and flourescein findings is long-standing. For the first time, we present histological evidence of a vascularised ERM associated with VPT. This raises additional key questions about the aetiology of ERM and the pathogenesis of VPT.

\section{Case report}

A 34-year-old healthy post-partum female presented after a month of experiencing floaters and reduced vision in her left eye. Her unaided visual acuity was 6/4 OD and 6/24 OS.

Dilated fundoscopy of the left eye revealed a medium-sized vasoproliferative tumour (VPT), located in the inferotemporal periphery. Adjacent to the tumour were intra-retinal exudates and telangiectatic vessels, plus an overlying exudative retinal detachment (Figure 1a). The VPT was also associated with a vascular looking macular ERM. Fundus fluorescein angiography
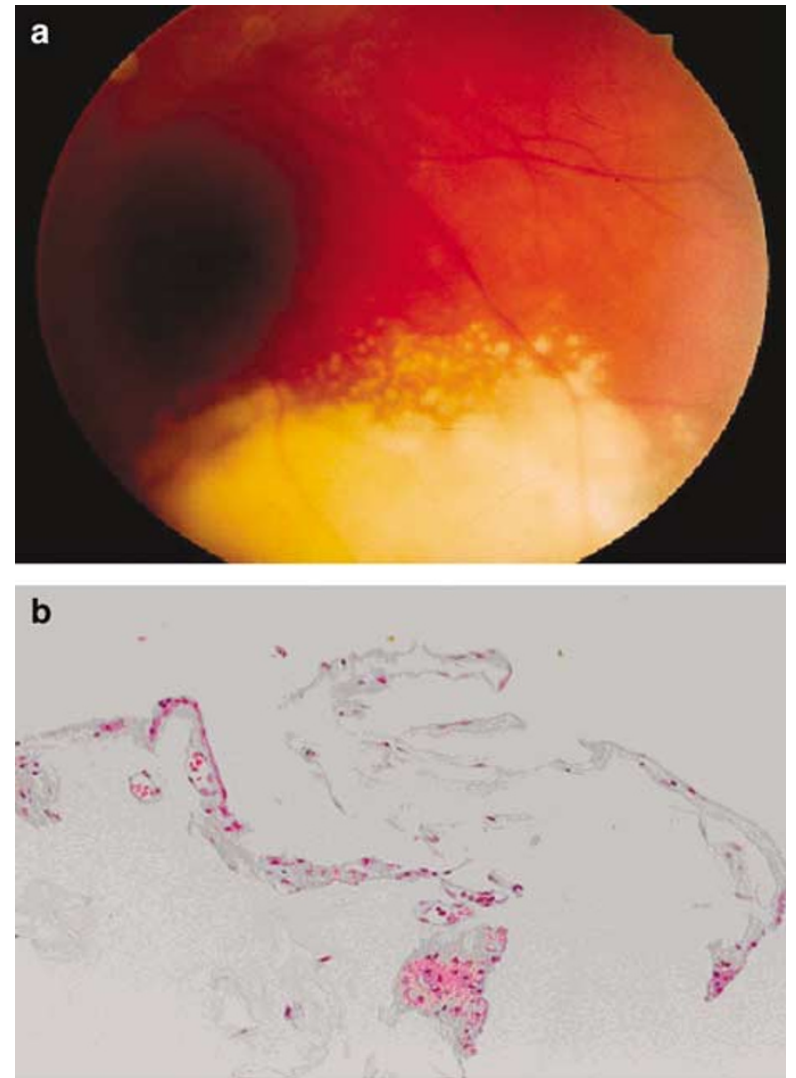

Figure 1 (a) Fundus photograph of the vasoproliferative tumour. (b) $\mathrm{H}+\mathrm{E}$ stain of the tumour-associated epiretinal membrane specimen at $\times 200$ magnification. Further studies revealed that the tissue comprised mainly of a hyaline membrane with positive-immunostaining for both type IV collagen and G-FAP (laminocytes). demonstrated late, generalised macular foveal capillary leakage.

Initially, she was treated with transconjunctival cryotherapy under local anaesthetic as she was once again pregnant. Visual acuity continued to deteriorate as a result of the ERM. On delivery a vitrectomy, membrane peel and more comprehensive cryotherapy to the VPT was undertaken under general anaesthetic (GA). The ERM was sent for histological examination. Small capillary vessels resembling diabetic neovascular membrane were present, confirming that the ERM associated with this VPT was also vascular in nature (Figure 1b).

\section{Comment}

Vascular ERM is predominantly a finding in proliferative diabetic retinopathy (PDR). ${ }^{1,2}$ In our Cambridge histopathological study of 207 ERMs, the only other nonPDR vascular ERM was induced by radiotherapy for basal cell carcinoma. ${ }^{3}$ In PDR, it appears that neovascularisation is a primary event, and glial tissue proliferation a secondary phenomenon. ${ }^{4}$

The aetiology of vascular ERM in VPT remains to be more clearly elucidated, raising further questions about the pathogenesis of both conditions. Apart from the vascular elements, the architecture and staining characteristics of this ERM was identical to nonvascularised ERM and posterior hyaloid membrane (PHM).$^{5}$ It is more-than-likely that the interplay of tumour-derived cytokines have a pivotal role in deciding the angiogenic status of VPT associated ERMs. ${ }^{6-8}$

The lack of previous reports of associated vascular ERM may be because of underdiagnosis of the condition.

\section{References}

1 Messmer EM, Heidenkummer HP, Kampik A. Ultrastructure of epiretinal membranes associated with macular holes. Graefes Arch Clin Exp Ophthalmol 1998; 236: 248-254.

2 Li K, Wong D, Hiscott P, Stanga P, Groenewald C, McGalliard J. Tryptan blue staining of internal limiting membrane and epiretinal membrane during vitrectomy visual results and histopathological findings. Br J Ophthalmol 2003; 87: 216-219.

3 Addison A, Snead D, Snead M. Radiation retinopathy secondary to radiotherapy for a lower lid BCC. Eye 2004; 18(12): 1273-1274.

4 Watanabe D, Suzuma K, Suzuma I, Ohashi H, Ojima T, Kurimoto $\mathrm{M}$ et al. Vitreous levels of angiopoietin 2 and vascular endothelial growth factor in patients with proliferative diabetic retinopathy. Am J Ophthalmol 2005; 139(3): 476-481.

5 Snead MP, Snead DR, Richards AJ, Harrison JB, Poulson AV, Morris AH et al. Clinical, histological and ultrastructural studies of the posterior hyaloid membrane. Eye (2002); 16(4): 447-453.

6 Rollin R, Mediero A, Martinez-Montero JC, Roldan-Pallares $\mathrm{M}$, Suarez-Leoz M, Vidal-Fernandez $\mathrm{P}$ et al. Atrial natriuretic peptide in the vitreous humor and epiretinal membranes of patients with proliferative diabetic retinopathy. Mol Vis 2004; 10: $450-457$.

7 Ioachim E, Stefaniotou M, Gorezis S, Tsanou E, Psilas K, Agnantis NJ. Immunohistochemical study of extracellular matrix components in epiretinal membranes of 
vitreoproliferative retinopathy and proliferative diabetic retinopathy. Eur J Ophthalmol 2005; 15(3): 384-391.

8 Schneeberger SA, Hjelmeland LM, Tucker RP, Morse LS. Vascular endothelial growth factor and fibroblast growth factor 5 are colocalized in vascular and avascular epiretinal membranes. Am J Ophthalmol 1997; 124(4): 447-454.

P Shankar'1, SE Bradshaw², A Ang ${ }^{1}$, IG Rennie ${ }^{3}$, DR Snead ${ }^{4}$ and MP Snead ${ }^{1}$

${ }^{1}$ VitreoRetinal Service, Addenbrooke's Hospital, Cambridge, UK

${ }^{2}$ Bristol Eye Hospital, Bristol, UK

${ }^{3}$ Department of Ophthalmology, Royal Hallamshire Hospital, Sheffield, UK

${ }^{4}$ Department of Pathology, Coventry and Warwick

University Hospitals, Coventry, UK

E-mail: pallavi_shankar@hotmail.com

No competing interests declared

Eye (2007) 21, 1003-1004; doi:10.1038/sj.eye.6702800; published online 6 April 2007

Sir,

Retinal pigment epithelial tear following

intravitreal injection of bevacizumab (avastin ${ }^{\circledR}$ ): optical coherence tomography and fluorescein angiographic findings

Bevacizumab is a humanised monoclonal antibody against all isoforms of vascular endothelial growth factor $^{1}$ (VEGF) for the treatment of choroidal neovascularisation.

We report a case with pigment epithelial detachment (PED) that developed a retinal pigment epithelial tear after intravitreal injection of bevacizumab.

This is, to our knowledge, the first report of this finding.

\section{Case report}

A 78-year-old fit patient presented with a 3 week history of decreased vision and metamorphopsia in her left eye. Best-corrected snellen visual acuity (VA) in this eye was $6 / 12$.

Fundoscopy showed subretinal fluid at the fovea with a large PED adjacent and inferior to it (Figure 1a). These changes were secondary to age-related macular degeneration (AMD). A fundus fluorescein angiogram (FFA) confirmed the presence of a subfoveal, minimally classic, choroidal neovascular membrane (CNVM) with a fibro vascular PED (Figure 1b).

Optical coherence tomography (OCT) scans confirmed the findings (Figure 1c). All scans were obtained with the Stratus ${ }^{\circledR}$ OCT scanner (Humphrey-Zeiss Inc., Dublin, CA, USA).

Photodynamic therapy (PDT) is contraindicated in the presence of a PED, ${ }^{2-5}$ and therefore the patient received an intravitreal injection of $2.5 \mathrm{mg}$ of bevacizumab (Avastin) with informed consent. Three weeks later left eye VA and metamorphopsia remained unchanged, although the patient had developed a retinal pigment epithelial tear inferior to the fovea (Figure 1d) and underwent FFA (Figure 1e) and OCT (Figure 1f).

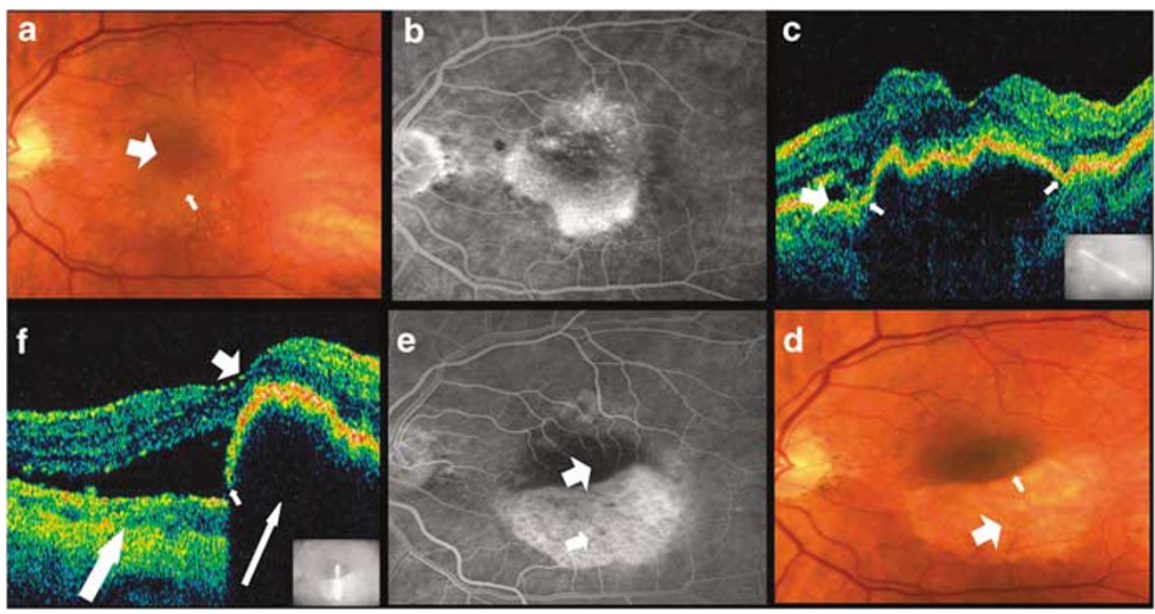

Figure 1 (a) Subretinal fluid at the fovea (hollow arrow) with a large PED adjacent and inferior to it (solid arrow). Best-corrected VA was $6 / 12$. (b) FFA $3 \mathrm{~min}$ and $16 \mathrm{~s}$ frame showed the presence of a subfoveal, minimally classic CNV with leakage and a fibrovascular PED. (c) A $3 \mathrm{~mm}$ single line OCT scan showing subretinal fluid (hollow arrow) and the extent of the PED (between solid arrows). (d) Retinal pigment epithelial tear inferior to the fovea after intravitreal injection of bevacizumab (2.5 mg). (Solid arrow indicates inwardly rolled edge of RPE. Hollow arrow indicates area denuded of RPE.) (e) FFA $21 \mathrm{~s}$ frame showed window defect corresponding to the area denuded of RPE (solid arrow) and hypofluorescence secondary blockage of choroidal fluorescence due to multilayered RPE (hollow arrow). (f) A $3 \mathrm{~mm}$ single line OCT scan showing fovea still apposed onto the RPE (short hollow arrow) and edge of inwardly rolled of RPE (short solid arrow) with resulting optical shadowing (long solid arrow) and increased reflectivity of outer band extent corresponding to the area denuded of RPE (long hollow arrow). VA remained unchanged as a result of the fovea remaining apposed onto the RPE despite the inferior to the fovea macular neuroretina being elevated and perpetuating the distortion of central vision. 